\title{
1 Intersubject consistent dynamic connectivity during natural vision revealed by functional MRI
}

3 Xin $\mathrm{Di}^{1,2}$, Bharat B Biswal $^{1,2 *}$

4

5 1. Department of Biomedical Engineering, New Jersey Institute of Technology, Newark, NJ, 07029, USA

6 2. School of Life Sciences and Technology, University of Electronic Science and Technology of China,

$7 \quad$ Chengdu, China

8

$9 *$ Corresponding author:

10 Bharat B. Biswal, PhD

11607 Fenster Hall, University Height

12 Newark, NJ, 07102, USA

13 bbiswal@yahoo.com

14 


\section{Abstract}

16 The functional communications between brain regions are thought to be dynamic. However, it is usually

17 difficult to elucidate whether the observed dynamic connectivity is functionally meaningful or simply due

18 to noise during unconstrained task conditions such as resting-state. During naturalistic conditions, such

19 as watching a movie, it has been shown that local brain activities, e.g. in the visual cortex, are consistent

20 across subjects. Following similar logic, we propose to study intersubject correlations of the time courses

21 of dynamic connectivity during naturalistic conditions to extract functionally meaningful dynamic

22 connectivity patterns. We analyzed a functional MRI (fMRI) dataset when the subjects watched a short

23 animated movie. We calculated dynamic connectivity by using sliding window technique, and quantified

24 the intersubject correlations of the time courses of dynamic connectivity. Although the time courses of

25 dynamic connectivity are thought to be noisier than the original signals, we found similar level of

26 intersubject correlations of dynamic connectivity to those of regional activity. Most importantly, highly

27 consistent dynamic connectivity could occur between regions that did not show high intersubject

28 correlations of regional activity, and between regions with little stable functional connectivity. The

29 analysis highlighted higher order brain regions such as the default mode network that dynamically

30 interacted with posterior visual regions during the movie watching, which may be associated with the

31 understanding of the movie.

32

33 Keywords: default mode network; dynamic connectivity; intersubject correlation; movie connectome;

34 naturalistic condition; supramarginal gyrus 


\section{Highlights}

37 Intersubject consistency may provide a complementary approach to study brain dynamic connectivity

- Widespread brain regions showed highly consistent dynamic connectivity during movie watching, while these regions themselves did not show highly consistent regional activity systems 


\section{Introduction}

45 The functional communications between spatially remote brain regions, especially the dynamics of connectivity, is a key to understand brain functions (Bullmore and Sporns, 2012; Friston, 2011; Park and

47 Friston, 2013). Recently, the dynamics of connectivity has drawn increasing interests of research,

48 especially in resting-state (Allen et al., 2014; Fu et al., 2019, 2018; Hutchison et al., 2013). However, due

49 to the unconstrained nature of resting-state, it is difficult to elucidate whether the observed changes of

50 connectivity across sliding windows are due to real fluctuations of functional communications, or simply

51 due to random fluctuations (Lindquist et al., 2014). Moreover, the blood-oxygen-level dependent (BOLD)

52 signals measured by fMRI are sensitive to physiological noises, such as respiration, heartbeat (Teichert et

53 al., 2010), and head motion (Power et al., 2012), which may give rise to spurious correlation estimates for

54 short window.

One way to capture meaningful dynamic functional connectivity is to manipulate subjects' mental states during the course of scan, so that there is known reference for the changes of connectivity. For example, in a typical task-based fMRI study with blocked design, different task conditions are assigned as blocks. Therefore, the time courses of dynamic connectivity can be correlated with the task design to identify task related connectivity changes (Di et al., 2015; Rosenthal et al., 2017). An alternative approach is to expose the subjects with naturalistic stimuli, such as a short movie. Although there is no

61 predefined references of dynamic connectivity changes, one may take advantage of the phenomenon of 62 intersubject correlation to capture changes that are consistent across different subjects (Hasson et al., 2004;

63 Nastase et al., 2019).

In the seminal study, Hasson and colleagues calculated intersubject correlations of the time series of BOLD signal (Figure 1A) when the subjects were watching a movie (Hasson et al., 2004). They demonstrated that several brain regions, especially the visual cortex, are highly correlated across subjects

67 during the movie watching. We propose that similar approach can be applied to the time courses of 68 dynamic connectivity to capture meaningful functional communication dynamics during natural vision.

69 Specifically, dynamic connectivity is usually calculated using a sliding window approach, so that a time 
series of dynamic connectivity can be obtained. The time courses of dynamic connectivity can then be

71 correlated across-subjects (Figure 1B). If the dynamic connectivity reflects real time functional

72 communications between regions that are caused by the viewing of natural stimuli, then the time courses

73 of dynamic connectivity from different subjects should somehow correlated. Therefore, we can apply

74 intersubject correlation method to identify meaningful dynamic communications between regions.

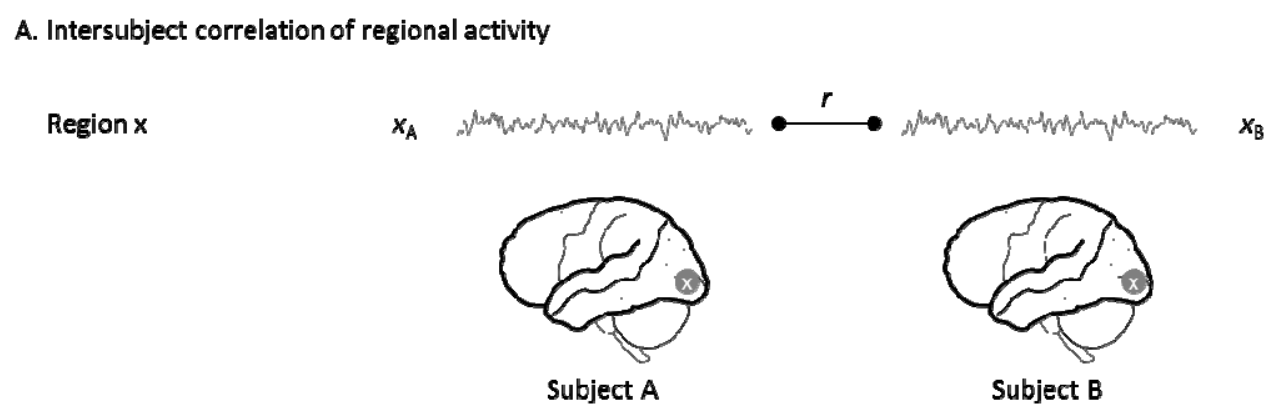

B. Intersubject correlation of dynamic connectivity

Dynamic connectivity

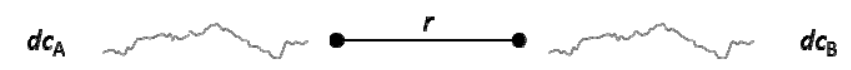

Sliding window

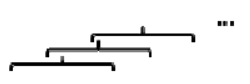

Region y

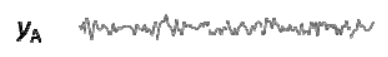

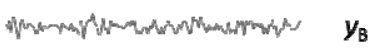

Region $\mathrm{x}$

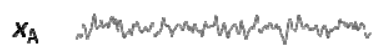

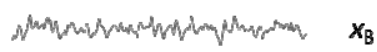

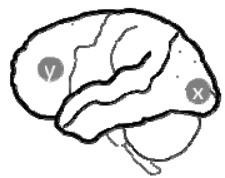

Subject A

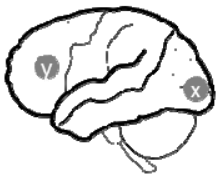

Subject B

Figure 1 Illustration of the calculations of intersubject correlations of the time series of regional activity (A) and the time courses of dynamic connectivity between two regions (B).

In the current study, we analyzed an fMRI dataset where the subjects were scanned when viewing a short animated movie. The aim was to identify dynamic connectivity that were shared cross subjects during the movie watching. In order to do so, we first performed regular intersubject correlation analysis to identify brain regions that showed consistent regional activity. Given these regions, we adopted a seed- 
83 based strategy to calculate dynamic connectivity between a seed region and every voxels in the brain. We

84 then evaluated and identified regions whose connectivity with the seed were consistent cross subjects.

85 Even though higher order association regions did not typically show high intersubject correlations of

86 regional activity (Hasson et al., 2004), their functional communications with lower order regions may be

87 consistent across subject following the narrative of the movie. We therefore hypothesized that

88 intersubject correlations of dynamic connectivity may be able to identify more widespread regions and

89 functional dynamics that are associated with the watching of the movie.

\section{Materials and methods}

\subsection{Data and task}

93 The fMRI data were obtained through openneuro (https://openneuro.org/; accession \#: ds000228). Only

94 the data from adult subjects were analyzed. There were originally 33 adult subjects. Two subjects' data were discarded because of poor brain coverage (subject \#: sub-pixar123 and sub-pixar124), and two were discarded due to large head motions (sub-pixar149 and sub-pixar150). As a result, a total of 29 subjects were included in the current analysis (17 females). The mean age is 24.6 years old (18 to 39 years).

99 Cloudy", which is 5.6 minutes long (https://www.pixar.com/partly-cloudy\#partly-cloudy-1). Brain MRI sagittal slices with $1 \square \mathrm{mm}$ isotropic voxels (GRAPPA parallel imaging, acceleration factor of 3; FOV:

$107256 \square \mathrm{mm}$ ). For more information about the dataset please refers to (Richardson et al., 2018).

\subsection{FMRI data analysis}




\subsubsection{Preprocessing}

FMRI data processing and analyses were performed using SPM12 and MATLAB (R2017b) scripts. A subject's T1 weighted structural image was first segmented into gray matter, white matter, cerebrospinal fluid, and other tissue types, and was normalized into standard Montreal Neurological Institute (MNI) space. The T1 images were then skull stripped based on the segmentation results. Next, all the functional images of a subject were realigned to the first image of the session and coregistered to the skull stripped T1 image of the same subject. Framewise displacement was calculated for the translation and rotation directions for each subject (Di and Biswal, 2015). Subjects who had maximum framewise displacement greater than $1.5 \mathrm{~mm}$ or $1.5^{\circ}$ were discarded from further analysis. See supplementary materials section $\mathrm{S} 1$ for additional analysis on the head motion effects. The functional images were then normalized to MNI space using the parameters obtained from the segmentation step with resampled voxel size of $3 \times 3 \times 3$ $\mathrm{mm}^{3}$. The functional images were then spatially smoothed using a Gaussian kernel of $8 \mathrm{~mm}$. Lastly, a voxel-wise general linear model (GLM) was built for each subject to model head motion effects (Friston's 24-parameter model) (Friston et al., 1996), low frequency drift (1/128 Hz), and constant offset. The residuals of the GLM were saved as a 4-D image series, which were used for further intersubject correlation analysis. The residual time series were all mean centered because of the constant term included in the GLM.

Removing low frequency drifts in BOLD signals is a critical step for dynamic connectivity analysis (Leonardi and Van De Ville, 2015). Leonardi and Van De Ville have suggested a high-pass filter of $1 / \mathrm{W} \mathrm{Hz}$ to avoid spurious dynamic connectivity caused by low-frequency fluctuations, where W represents the window length in the sliding window analysis. The high-pass filter of $1 / 128 \mathrm{~Hz}$ is the default in the GLM module in SPM. Given the window length of 60 s (30 TR) in the current analysis, we also applied high-pass filtering of $1 / 64 \mathrm{~Hz}$ in a supplementary analysis. The results are very similar to what using the $1 / 128 \mathrm{~Hz}$ high-pass filtering (see supplementary materials section S3).

\subsubsection{Intersubject correlation analysis}


134 The correlations of time series of either brain activity or dynamic connectivity are calculated between 135 pairs of subjects. If there are $\mathrm{N}$ subjects, then there will be $\mathrm{N} x(\mathrm{~N}-1) / 2$ correlation coefficients. The 136 statistics of these correlations become tricky, because they are calculated from only $\mathrm{N}$ subjects, therefore 137 not independent. An alternative approach is leave-one-out (Nastase et al., 2019), where the time series of 138 one hold-out subject were correlated with the averaged time series of the remaining $\mathrm{N}-1$ subjects. The 139 averaged time series of $\mathrm{N}-1$ subjects were thought to reflect the consistent component rather than noisy 140 individual's time series. Therefore, the resulting correlations should be higher than the pair-wise 141 correlations. Another benefit is that this approach estimates one correlation for each subject, making 142 group level statistics easier. Therefore, we adopt the leave-one-out approach in the current analysis. We first performed intersubject correlation analysis on regional activity time series. The preprocessed BOLD time series were extracted for each voxel and subject in a gray matter mask. For a given voxel, the time series of one subject was held out, and the averaged time series of the remaining subject were calculated. Then the time series of the hold-out subject were correlated with the averaged

147 time series. This process was performed for every voxel and every subject, resulting in one correlation map for one subject. The correlation maps were transformed into Fisher's z maps. Group level one sample t test was then performed to identify regions whose intersubject correlations were consistently greater than 0 . However, the null hypothesis statistical significance testing may not provide much information of the effect size. There may be only small but consistent correlations for each subject, which could give rise to very high statistical significance in a one sample t test. Indeed, when doing such null hypothesis statistical significance testing for intersubject correlation analysis, usually almost all the brain regions will show somehow significant correlations (Chen et al., 2016). We are more interested and focused on the real effect size, i.e. correlation coefficients, in our analysis. We therefore averaged the Fisher's z maps, and transformed them back into $r$ maps. The continuous $r$ maps were shown in the 157 results section. approach. Given that a set of brain regions showed high intersubject correlations of regional activity, we 
160

161

162

163

164

165

166

167

defined these regions as seeds. We adopted a relatively high threshold of $r>0.45$ for the averaged intersubject correlation map of regional activity to isolate four visual related seeds. Two of them were located in the medial and posterior portion of the occipital lobe, which mainly covered the lingual gyrus and calcarine sulcus. The other two seeds were located bilaterally in the middle occipital gyrus and extended to the middle temporal gyrus. We labeled them as left and right medial visual and lateral visual seeds, respectively. In addition, we adopted a relatively low threshold of $r>0.35$ to isolate the left and right supramarginal gyrus seeds. The maps of the six seeds are available at:

https://identifiers.org/neurovault.collection:6245.

For each seed, we performed voxel-wise correlation analysis, i.e. calculating intersubject correlations of dynamic connectivity between the seed and every voxel in the gray matter mask. For two given time series from a seed and a voxel, we first applied sliding window technique to calculate dynamic connectivity for each subject. The window length was set as 30 time points (60 s) (Nastase et al., 2019), and the time step was set as 2 time point (4s). Therefore, the time course of dynamic connectivity had 70 window steps. Next, we calculated correlations between the time courses of dynamic connectivity of a given subject with the averaged dynamic connectivity of the remaining subjects for a given voxel. As a result, there was one correlation map for each seed and each subject.

The $r$ maps of correlations of dynamic connectivity were transformed into Fisher's z maps for group level statistical analysis. Again, we simply calculated an averaged z map for a seed, and transformed it back into $\mathrm{r}$ map. In addition, we performed group-level analysis to identify regions that showed different dynamic connectivity patterns with different levels of seeds. Specifically, we calculated contrast images from the Fisher's z maps for each subject representing the differences between specific levels of seeds compared with the other seeds. For example, we calculated a contrast image using [1, 1, $0.5,-0.5,-0.5,-0.5]$ on the six z maps of a subject to define the differences between the two medial visual seeds and the remaining four seeds. The contrast images were entered into a one sample t test GLM using nonparametric statistics in SnPM13 (Statistical NonParametric Mapping, http://warwick.ac.uk/snpm). Resulting clusters were first formed at $\mathrm{p}<0.001$, and the cluster extend was thresholded using family- 
186

187

188

189

190

191

192

193

194

195

196

197

198

199

200

201

202

203

204

205

206

207

208

209

210

211

wise error (FWE) corrected $\mathrm{p}<0.0167(0.05 / 3)$. The cluster level FWE threshold $(0.0167)$ was chosen

to further account for the three levels of seeds (medial visual, lateral visual, and supramarginal seeds).

In addition to the voxel-based analysis, we also performed region of interest (ROI)-based analysis

for in-depth examinations of the dynamic connectivity effects. In addition to the six seeds, we included

three more regions that showed different intersubject correlations of dynamic connectivity with different

seeds. Specifically, they were the left precentral gyrus that showed higher intersubject correlations of

dynamic connectivity with the medial visual seeds, and the posterior cingulate cortex and medial

prefrontal cortex that showed higher intersubject correlations of dynamic connectivity with the

supramarginal gyrus seeds. The regions were defined based on the statistical significant clusters from the group-level analysis. The maps of the three regions are available at:

https://identifiers.org/neurovault.collection:6245. The calculations of intersubject correlations of dynamic connectivity were the same as the seed-based analysis.

The selections of sliding window length is nontrivial (Fu et al., 2014; Zhang et al., 2013). In

addition to the 30-TR window length, we also explored other window length of 10 TRs (20 s), 20 TRs (40

s), 40 TRs (80 s), 50 TRs (100 s), and 60 TRs (120 s). For each window length, we calculated

intersubject correlations of dynamic connectivity among the 9 ROIs.

\subsubsection{Relations with other measures}

We first compared the intersubject consistent dynamic connectivity with stable functional connectivity.

For each subject, we calculated correlation coefficients across the 9 ROIs, and transformed them into

Fisher's z. Then the z matrices were averaged across the 29 subjects, and transformed back into r values.

In addition, we calculated the consistent component of each ROI, i.e. averaging the time series across the 29 subjects. And then one single correlation matrix among the 9 ROIs was calculated.

Given the consistent component of the 9 ROIs, we also calculated dynamic connectivity between pairs of ROIs using the same sliding window approach. The time courses of dynamic connectivity calculated from the consistent component were compared with the averaged dynamic connectivity that was calculated from each subject. 
213 by the consistent component of regional activity, or by the subject-specific idiosyncratic component. To

214 do so, for each ROI, we regressed out the consistent component from each subject's time series, and

215 calculated dynamic connectivity from the residual time series for each subject. Intersubject correlations

216 of dynamic connectivity calculated from the residual time series were compared with those from the taw

217 time series.

218 Lastly, we calculated intersubject correlations of regional activity using the same sliding window 219 approach for the 9 ROIs. That is, for each ROI, intersubject correlation was calculated at each window, 220 resulting in a time course of intersubject consistency of regional activity in each of the ROI.

\section{3. Results}

\section{3.1. Intersubject correlations of regional activity}

224 We first calculated intersubject correlations of regional activity for every voxel in the brain during the 225 video watching (Figure 2A). The highest correlations were around 0.5. The major regions that had high 226 intersubject correlations were the visual cortex extending anterior to the fusiform gyrus and middle 227 temporal lobe. The bilateral supramarginal gyrus also showed high intersubject correlations. The 228 bilateral precentral gyrus also showed intersubject correlations, but the effect sizes were much smaller.

229 Figure 2A shows all the voxels with positive correlation values. It is noteworthy that many regions

230 showed very small intersubject correlations, including largely the prefrontal cortex and anterior temporal 231 lobe. 


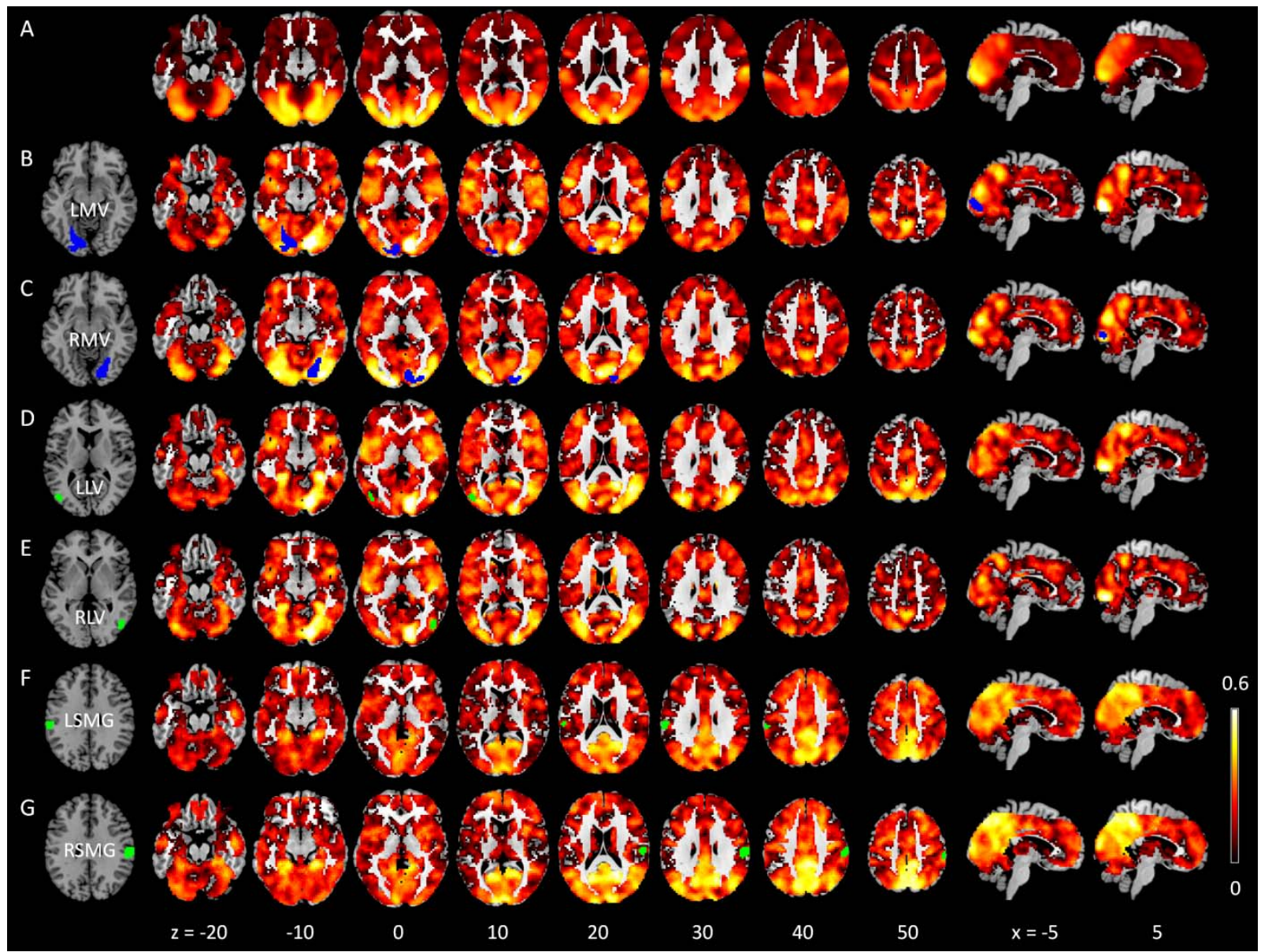

233 Figure 2 Intersubject correlation maps of regional activity (A) and dynamic connectivity with different

234 seeds (B through G). The seed regions were depicted in blue or green in respective rows. All voxels with

235 positive correlations are shown. The numbers at the bottom represent $\mathrm{z}$ or $\mathrm{x}$ coordinates in Montreal

236 Neurological Institute (MNI) space. LMV, left medial visual; RMV, right medial visual; LLV, left lateral

237 visual; RLV, right lateral visual; LSMG, left supramarginal gyrus; RSMG, right supramarginal gyrus. All

the maps are available at: https://identifiers.org/neurovault.collection:6245.

\subsection{Intersubject correlations of dynamic connectivity}

\section{$241 \quad 3.2 .1$ Seed-based analysis}

242 We defined seed regions where there were high intersubject correlations of regional activity, which

243 included bilateral medial visual regions, lateral visual regions, and supramarginal gyrus. We next 
calculated voxel-wise intersubject correlations of dynamic connectivity with the six seeds, respectively

245 (Figure 2B through 2G). There were widespread brain regions that showed intersubject consistent dynamic correlations with different seeds. First of all, the effect sizes of the intersubject correlations of

247 dynamic connectivity, i.e. the correlation coefficients, were comparable to those in the intersubject

248 correlations of regional activity. Secondly, regions with intersubject correlations of dynamic connectivity

249 turned out to be more widespread and extended to the frontal and parietal regions that did not show high

250 intersubject correlations of regional activity. See supplementary materials section S2 for direct

comparisons between the intersubject correlations of dynamic connectivity and those of regional activity.

252 Thirdly, the left and right corresponding seeds showed similar dynamic connectivity patterns, but there

253 were substantial differences in the patterns of dynamic connectivity among medial visual, lateral visual,

254 and supramarginal gyrus seeds. In order to highlight specific brain regions that showed dynamic

connectivity with different seeds, we compared each pair of seeds with the remaining seeds using

261 other seeds, which basically formed the default mode network.

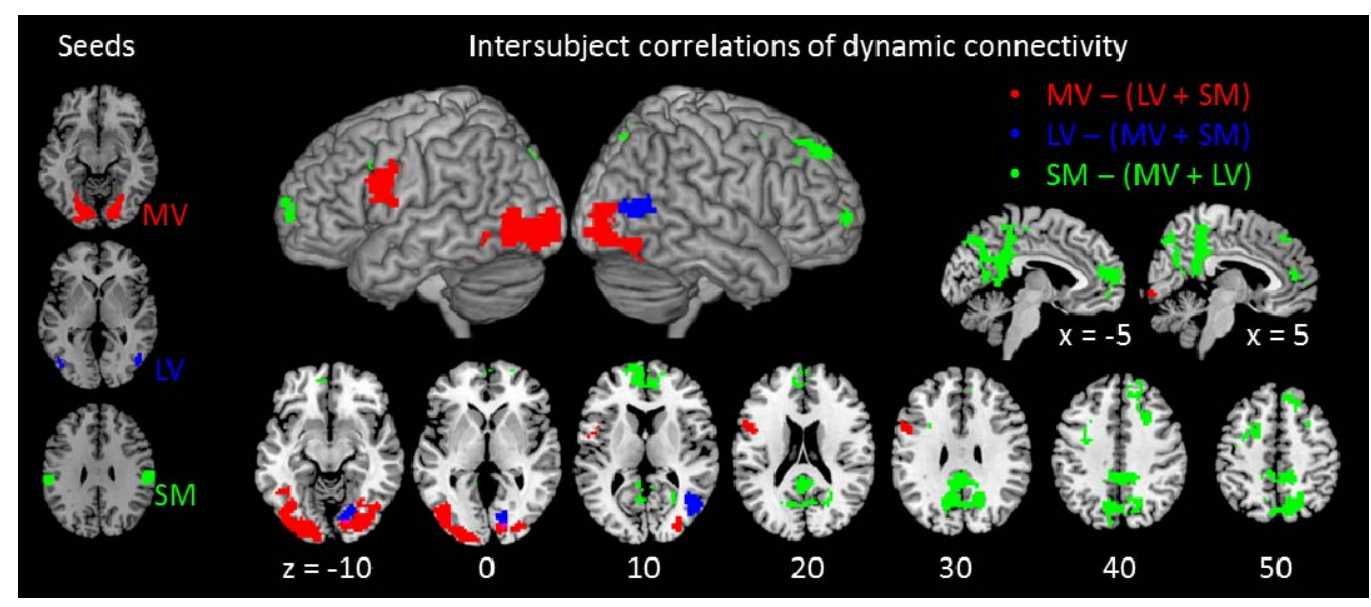


Figure 3 Differential intersubject correlations of dynamic connectivity among the medial visual, lateral visual, and supramarginal gyrus seeds (depicted on the left). All maps were thresholded at $\mathrm{p}<0.001$, and cluster thresholded at $\mathrm{p}<0.0167(0.05 / 3)$ after family-wise error $(\mathrm{FWE})$ correction using nonparametric method. MV, medial visual; LV, lateral visual; and SM, supramarginal gyrus. Unthresholded statistical maps are available at: https://identifiers.org/neurovault.collection:6245.

\subsubsection{Relations with stable functional connectivity}

In order to better understand and interpret the dynamic connectivity and regional functions, we further calculated different types of connectivity measures among a set of regions of interest. In addition to the six seeds, we defined left precentral gyrus, posterior cingulate cortex, and medial prefrontal cortex ROIs that showed different dynamic connectivity with different seeds. Among the 9 regions, we calculated regular mean functional connectivity (Figure 4A) and connectivity derived from the consistent components across the 29 subjects (Figure 4B). These two correlation matrices looked similar, and negative correlations with the association regions in the consistent component correlations.
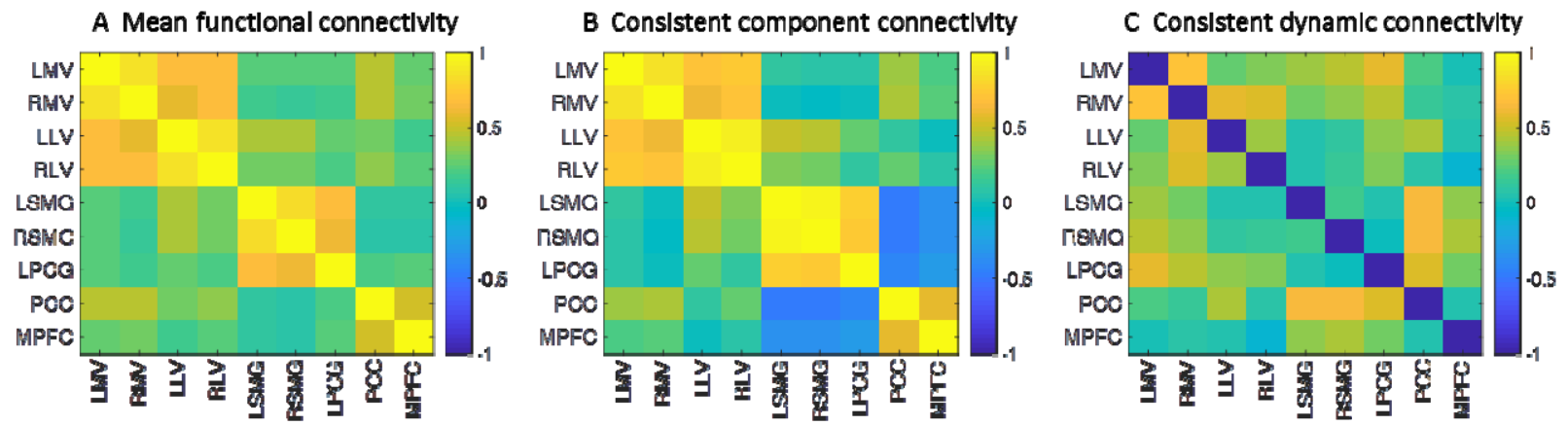

281 Figure 4 Correlation matrices among the 9 regions of interest (ROI) using different methods. A) Mean

282 functional connectivity across the 29 subjects. B) Correlations of the consistent component of each ROI 283 (averaged time series across the 29 subjects). C) Intersubject correlations of dynamic connectivity. LMV, 
left medial visual; RMV, right medial visual; LLV, left lateral visual; RLV, right lateral visual; LSMG,

left supramarginal gyrus; RSMG, right supramarginal gyrus; LPCG, left precentral gyrus; PCC, posterior cingulate cortex; and MPFC, medial prefrontal cortex.

The intersubject consistent dynamic connectivity matrix (Figure 4C) was largely different from

the two stable correlation matrices. Some high consistent dynamic connectivity was observed within the

visual regions. The highest correlation was between the left and right medial visual regions $(r=0.70)$. In

contrast, many consistent dynamic connectivity were shown between different functional networks, where

there were virtually none or even negative stable correlations. Specifically, the medial visual regions

293 showed high consistent dynamic connectivity with the left precentral gyrus ROI. The highest intersubject

294 correlation was 0.56 between left medial visual region and left precentral gyrus. The default mode

295 regions and supramarginal regions also showed high consistent dynamic connectivity. The highest

correlation was 0.64 between the posterior cingulate cortex and right supramarginal gyrus. It is

noteworthy that these regions generally showed negative stable correlations in Figure 4B. course. 

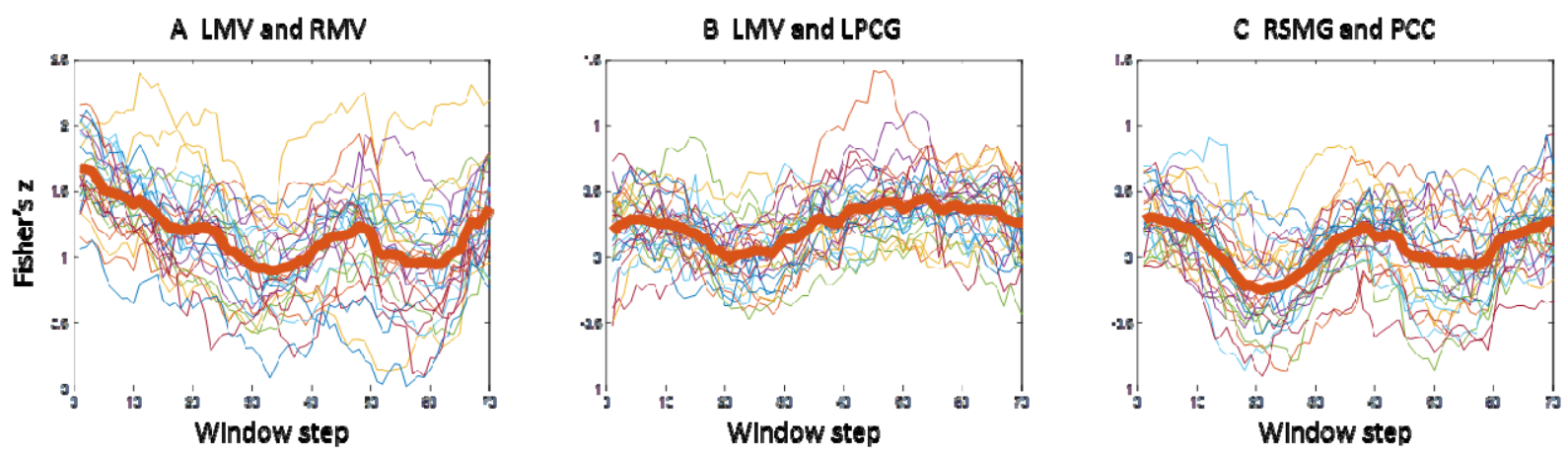

Figure 5 Time courses of dynamic connectivity (Fisher's z) for three pairs of regions of interest. Each

thinner line represents the time course of one subject, and the thicker red lines represent the averaged time

\subsubsection{Relations with the consistent component}

A subsequent question is that whether the observed intersubject consistent dynamic connectivity is driven by the consistent component of regional activity across subject, or by subject-specific idiosyncratic components. We then regressed out the consistent component for each subject's time series and calculated intersubject correlations of dynamic connectivity from the residual time series (Figure 6B). Compared with the intersubject correlations of dynamic connectivity from the original analysis (Figure

$3216 \mathrm{~A}$ ), the consistency of dynamic connectivity from the residual time series were largely diminished.

322 Figure 6D and 6E illustrate the changes of dynamic connectivity time courses after the regression

323 between a representative ROI pair, i.e. right supramarginal gyrus and posterior cingulate cortex (see supplementary Figure S4 for other ROI pairs). The intersubject correlation reduced from 0.64 to 0.29 . Figure $6 \mathrm{C}$ illustrates the dynamic connectivity of the consistent components of regional activity between these two ROIs. The fluctuating pattern was similar to those calculated from individual subject's original time series (Figure 6D), further confirmed that the consistent dynamic connectivity across individuals was 

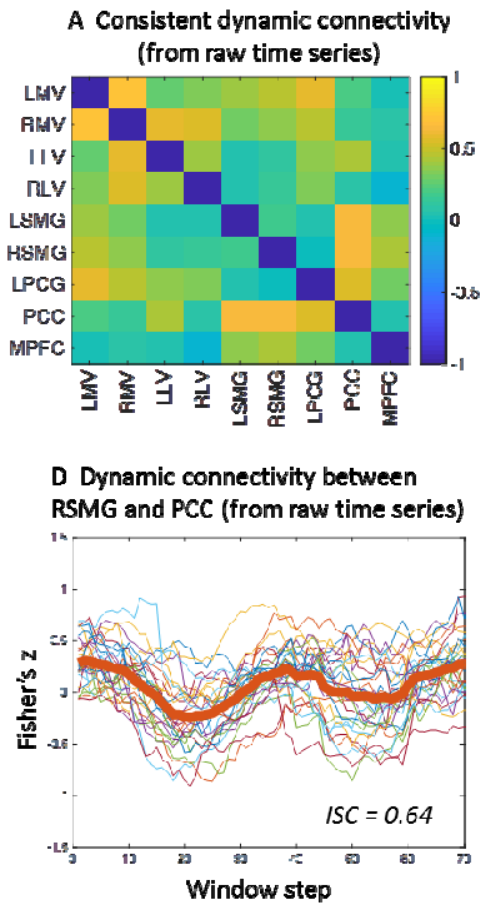

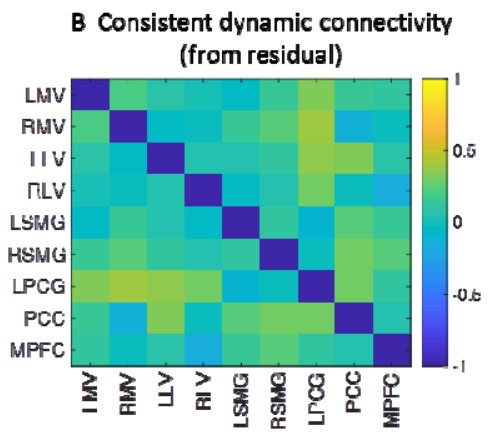

E Dynamic connectivity between RSMG and PCC (from residual)

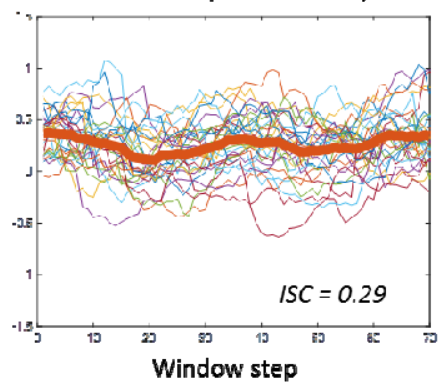

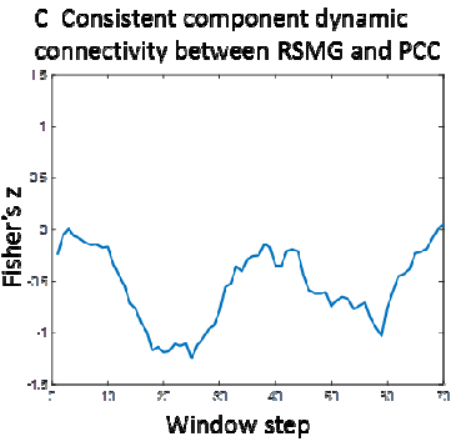

F Dynamic intersubject correlations of regional activity

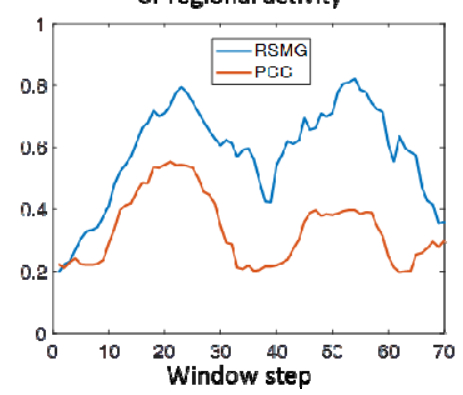

Figure 6 A) and B) Intersubject correlations (ISC) of dynamic connectivity calculated from raw time series (A) and residual time series after regressing out the intersubject consistent components (B). C)

Dynamic connectivity of the consistent component of regional activity between right supramarginal gyrus (RSMG) and posterior cingulate cortex (PCC). D) and E) Time courses of dynamic connectivity (Fisher's z) between RSMG and PCC calculated from raw time series (D) and the residual time series after regressing out the intersubject consistent component. F) The time courses of intersubject correlations of regional activity in RSMG and PCC.

(supplementary Figure S5). Figure 6F shows the time courses of intersubject correlations of regional activity in the right supramaginal gyrus and posterior cingulate cortex ROIs. Both regions showed similarly but reversed time courses as the dynamic connectivity between them. That is, during the two periods of dips of dynamic connectivity, there were elevated intersubject correlations of regional activity in both regions. But this kind of close relations cannot be observed in the other two pairs of ROIs (supplementary Figure S4). 


\subsubsection{Effects of sliding-window length}

346 We repeated the ROI-based intersubject correlation analysis of dynamic connectivity using different

347 window length from 10 TRs to 60 TRs. The intersubject correlation matrices were in general weaker

348 when the window was shorter, especially for the 10-TR window (Figure 7). As the window went longer,

349 the correlations matrices became similar to the 30-TR window results. But for even longer window, there

350 were two different trends. First, some of the intersubject correlations kept increasing, usually between

351 regions that involved in one or two visual ROIs (Figure 7B). On the other hand, some of the intersubject

352 correlations decreased after peaked at the 30-TR window, usually between regions that involved

353 supramarginal gyrus or posterior cingulate cortex. Figure 7C illustrated the time courses of dynamic

354 connectivity between right supramarginal gyrus and posterior cingulate cortex. It can be seen that the

355 variability of dynamic connectivity time courses were larger in short window. When using 10-TR

356 window, the dynamic connectivity changed fast, and were not aligned across subjects. When using 30-

357 TR window, the dynamic connectivity time courses became smoother, and the fluctuations were more

358 aligned across subjects, which in turn gave rise to higher intersubject correlations. But when using 50-TR

359 window, the time courses of dynamic connectivity become too smooth, so that the subject averaged trend

360 become less apparent. It's noteworthy that for the dynamic connectivity between the left and right medial

361 visual ROIs and between left medial visual and left precentral gyrus ROIs, there were linear trends of

362 dynamic connectivity across subjects, which gave rise to high intersubject correlations in longer windows

363 (Figure S6). 

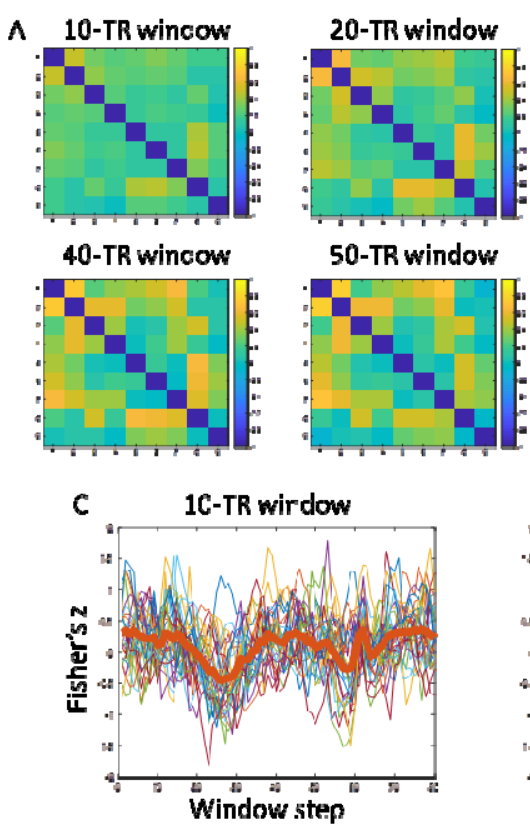
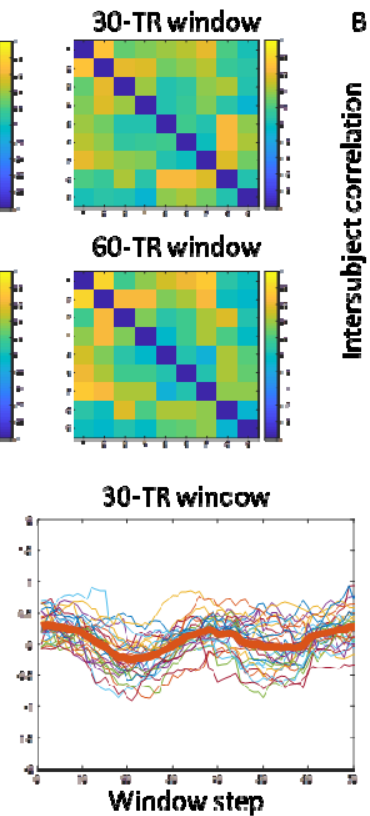
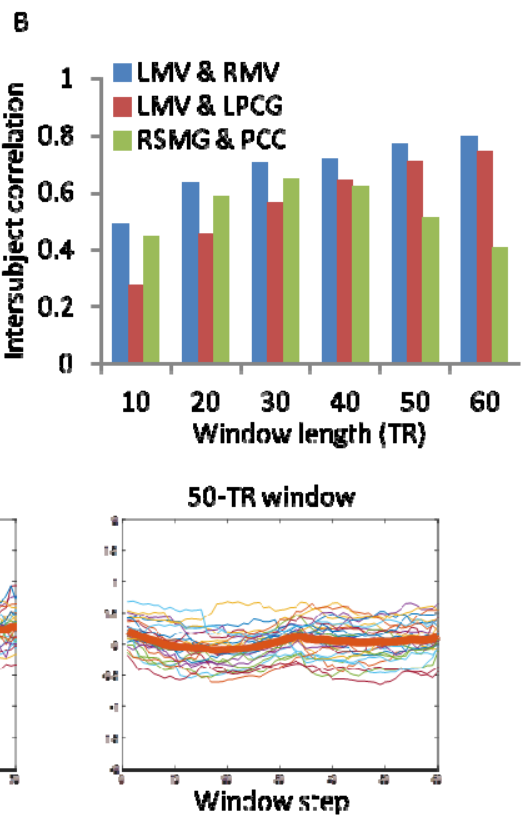

Figure 7 A) The effects of sliding-window length on the intersubject correlations of dynamic connectivity.

B) Intersubject correlations of dynamic connectivity of three pairs of regions of interest: left medial visual

$$
\text { gyrus (RSMG) and posterior cingulate cortex (PCC). C) The time courses of dynamic connectivity }
$$
between RSMG and PCC in three window lengths. TR, repetition time.

\section{Discussion}

373 In the current study, we proposed intersubject correlation analysis on the time courses of dynamic

374 connectivity during natural vision. We were able to identify intersubject consistent dynamic connectivity

375 at similar levels as the intersubject correlations of regional activity, although the time courses of dynamic

376 connectivity were thought to be nosier than the original time series. By using seed regions from the

377 visual cortex and supramarginal gyrus, we demonstrated widespread brain regions that showed high

378 intersubject consistent dynamic connectivity with these seeds, although these regions themselves did not

379 show intersubject correlations of regional activity. These regions included high order association regions

380 such as frontal and parietal regions, as well as the default mode network. The intersubject consistent 
patterns of dynamic connectivity support the functional meaningfulness of dynamic connectivity during movie watching, and suggest that dynamic connectivity could be a complementary avenue to characterize the functions of a brain region.

The brain regions that had the highest intersubject correlations of regional activity are mainly in the posterior visual related regions, which are consistent with previous studies (Hasson et al., 2004; Nummenmaa et al., 2012). In addition, the current study found dynamic connectivity among different levels of visual areas that were highly consistent across subjects. This is interesting because although the overall functional connectivity among the visual areas are very high (Figure 4A), there are still functionally meaningful fluctuations of interactions among the visual regions. The observable dynamics of connectivity among visual areas are in line with previous studies showing task modulated connectivity among visual areas in different task conditions (Di et al., 2019, 2015; Di and Biswal, 2017). It is interesting to note that the dynamics of intersubject correlations of regional activity in the visual areas also showed decreased trends at the beginning of the session (Figure S4 and S5). Therefore, the decreased connectivity in the beginning may reflect adaptations effects in the visual areas. However, during the latter half of the session, the dynamics of intersubject correlations of regional activity kept at a stable level, which cannot explain the dynamics of connectivity between them (Figure S4). probably due to different movies the participants watched. Given their critical role in empathy (Silani et al., 2013), it is reasonable to observe high intersubject correlations in the supramarginal gyrus during the watching of the animated movie, which involves the understanding the intentions of different animated characters. Interestingly, the intersubject correlations of regional activity in the supramarginal gyrus also

404 showed dynamics, with two periods of high correlations roughly between the $20^{\text {th }}$ and $30^{\text {th }}$ windows and 405 between the $50^{\text {th }}$ and $60^{\text {th }}$ windows (Figure 6F and S4B). The first may correspond to the scene when 406 Peck the stork and Gus the cloud first met, where their interactions appeared to be different from the other 
407

408

409

410

411

412

413

414

415

416

417

418

419

420

421

422

423

424

425

426

427

428

429

430

431

432

storks and clouds. The second may coincide with the scene when Peck flew away, and Gus thought Peck had abandoned him. These scenes require active inferences of the intentions of the characters, and may involve mismatches between predictions and the actual story development. Therefore, it is reasonable to see high cross-subject similarities in the supramarginal gyrus during these two periods.

In addition to regional activity, we also found that the default mode network showed highly consistent dynamic connectivity with the supramarginal gyrus regions. Similar to the supramarginal gyrus ROIs, the regional activity in the posterior cingulate cortex showed two periods of high consistent regional activity (Figure 6F). But interestingly, during these two periods the posterior cingulate cortex and supramarginal gyrus showed strong anti-correlation (Figure 6C). The default mode network involves high-order representation of the world (Carhart-Harris and Friston, 2010). And the functional communications between the default mode network and supramarginal gyrus may reflect the prediction error between inner representation and the input from the video. Similar to a previous study using dynamic intersubject connectivity analysis (Simony et al., 2016), both of the studies highlighted the critical role of the default mode network in understanding of the narratives of a movie.

Generally speaking, the intersubject consistent connectivity and stable functional connectivity showed disassociations. Specifically, the ROI pairs that showed highly consistent dynamic connectivity may have high stable functional connectivity or very low overall connectivity. The latter case may be more interesting, because it suggests transient functional communications that cannot be observed in traditional functional connectivity analysis. The 9 ROIs used in the current analysis are from three functional modules, i.g. unimodal visual, higher order task positive, and default mode networks. The three functional modules can be confirmed in the matrix of stable connectivity (Figure 4A), where there are high within-module functional connectivity but weak between-module connectivity. The matrix of consistent dynamic connectivity, on the other hand, showed that there are more between-module dynamic connectivity. These results are in line with the economy account of brain network organizations, which suggests that the functional communications between modulates are costly in terms of energy consumption, therefore are more transient (Bullmore and Sporns, 2012). It is also consistent with the 
findings that the connectivity between modules are more variable and context dependent (Di and Biswal, 2019; Fu et al., 2017).

By calculating dynamic connectivity time courses from individual's time series, the proposed method focused on the consistency of the dynamic connectivity time courses across subjects. Our

437 additional analysis showed that the consistent dynamic connectivity time courses was driven by the

438 dynamic connectivity of the consistent component of the regional time series, at least for the current video

439 watched. The latter method provides a simple approach to reveal the dynamics of connectivity, and is

440 closely related to the dynamic intersubject functional connectivity approach proposed by Simony et al.

441 (Simony et al., 2016). Our method, on the other hand, can not only reveal the time course of dynamic

442 connectivity, but can also provide a quantity of a connection about how the dynamic connectivity is

443 consistent across subjects. Eventually, we will be able to obtain a matrix of the consistency of dynamic

444 connectivity among ROIs from the whole brain. This is important because the seed-based approach used

445 in the current analysis may miss dynamic connectivity between regions that do not have consistent

446 regional activity. The connectome-based approach can provide a comprehensive mapping of dynamic

447 communications across the brain during the watching of a movie, and can be seen a special form of task connectome (Di and Biswal, 2019).

The selection of window length for dynamic connectivity analysis is nontrivial (Fu et al., 2014; connectivity could be. However, less time points for each window would also mean noisier estimates of connectivity. In the current analysis, 10-TR window gave very noisy estimate of functional connectivity, thus making intersubject correlations very low. On the other hand, longer window will make connectivity 454 estimate accurate, but at a cost of losing temporal resolution. In the context of the current video watched, 455 30-TR window seems a balance. However, this time scale of dynamic connectivity fluctuations may not 456 be easily generalized to other videos or to resting-state. But it certainly can provide some insight to the 457 chosen of window length in future studies. In addition, some computational method may be used to avoid 458 the window length issue, e.g. using adaptive covariance estimates (Fu et al., 2014; Zhang et al., 2013) or 
459

460

461

462

463

464

465

466

467

468

469

470

471

472

473

\section{Conflict of interest}

475

476

477

478

479

480

481

482

483

484

485

486

487

488 (Glerean et al., 2012).

\section{Conclusion}

\section{Acknowledgement:}

DA038895).

\section{References:}

window-free method such as Kalman filtering (Kang et al., 2011) or instantaneous phase synchronization

In the current study, we proposed intersubject correlation analysis on dynamic connectivity. The results revealed widespread brain regions that showed consistent intersubject correlations of dynamic connectivity. The consistent correlations support the functional significance of dynamic connectivity during natural vision. The method may provide a complementary approach to understand the dynamic nature of brain functional integrations.

This study was supported by grants from (US) National Institute of Health (R01 AT009829; R01

The authors declared that there is no conflict of interest.

Allen, E.A., Damaraju, E., Plis, S.M., Erhardt, E.B., Eichele, T., Calhoun, V.D., 2014. Tracking wholebrain connectivity dynamics in the resting state. Cereb. Cortex N. Y. N 1991 24, 663-76. https://doi.org/10.1093/cercor/bhs352

Bullmore, E., Sporns, O., 2012. The economy of brain network organization. Nat. Rev. Neurosci. 13, 336-349. https://doi.org/10.1038/nrn3214

Carhart-Harris, R.L., Friston, K.J., 2010. The default-mode, ego-functions and free-energy: a neurobiological account of Freudian ideas. Brain J. Neurol. 133, 1265-83. https://doi.org/10.1093/brain/awq010

Chen, G., Shin, Y.-W., Taylor, P.A., Glen, D.R., Reynolds, R.C., Israel, R.B., Cox, R.W., 2016. Untangling the relatedness among correlations, part I: Nonparametric approaches to inter-subject 
correlation analysis at the group level. NeuroImage 142, 248-259. https://doi.org/10.1016/j.neuroimage.2016.05.023

Di, X., Biswal, B.B., 2019. Toward Task Connectomics: Examining Whole-Brain Task Modulated Connectivity in Different Task Domains. Cereb. Cortex 29, 1572-1583. https://doi.org/10.1093/cercor/bhy055

Di, X., Biswal, B.B., 2017. Psychophysiological Interactions in a Visual Checkerboard Task: Reproducibility, Reliability, and the Effects of Deconvolution. Front Neurosci 1-36. https://doi.org/10.3389/fnins.2017.00573

Di, X., Biswal, B.B., 2015. Characterizations of resting-state modulatory interactions in the human brain. J. Neurophysiol. 114, 2785-96. https://doi.org/10.1152/jn.00893.2014

Di, X., Fu, Z., Chan, S.C., Hung, Y.S., Biswal, B.B., Zhang, Z., 2015. Task-related functional connectivity dynamics in a block-designed visual experiment. Front. Hum. Neurosci. 9, 1-11. https://doi.org/10.3389/fnhum.2015.00543

Di, X., Zhang, Z., Biswal, B.B., 2019. Understanding psychophysiological interaction and its relations to beta series correlation. bioRxiv 322073. https://doi.org/10.1101/322073

Fox, M.D., Snyder, A.Z., Vincent, J.L., Corbetta, M., Van Essen, D.C., Raichle, M.E., 2005. The human brain is intrinsically organized into dynamic, anticorrelated functional networks. Proc. Natl. Acad. Sci. U. S. A. 102, 9673-8.

Friston, K.J., 2011. Functional and effective connectivity: a review. Brain Connect. 1, 13-36. https://doi.org/10.1089/brain.2011.0008

Friston, K.J., Williams, S., Howard, R., Frackowiak, R.S., Turner, R., 1996. Movement-related effects in fMRI time-series. Magn. Reson. Med. Off. J. Soc. Magn. Reson. Med. Soc. Magn. Reson. Med. 35, 346-55. https://doi.org/DOI 10.1002/mrm.1910350312

Fu, Z., Chan, S.-C., Di, X., Biswal, B., Zhang, Z., 2014. Adaptive covariance estimation of non-stationary processes and its application to infer dynamic connectivity from fMRI. IEEE Trans. Biomed. Circuits Syst. 8, 228-39. https://doi.org/10.1109/TBCAS.2014.2306732

Fu, Z., Tu, Y., Di, X., Biswal, B.B., Calhoun, V.D., Zhang, Z., 2017. Associations between Functional Connectivity Dynamics and BOLD Dynamics Are Heterogeneous Across Brain Networks. Front. Hum. Neurosci. 11. https://doi.org/10.3389/fnhum.2017.00593

Fu, Z., Tu, Y., Di, X., Du, Y., Pearlson, G.D., Turner, J.A., Biswal, B.B., Zhang, Z., Calhoun, V.D., 2018. Characterizing dynamic amplitude of low-frequency fluctuation and its relationship with dynamic functional connectivity: An application to schizophrenia. NeuroImage 180, 619-631. https://doi.org/10.1016/j.neuroimage.2017.09.035

Fu, Z., Tu, Y., Di, X., Du, Y., Sui, J., Biswal, B.B., Zhang, Z., de Lacy, N., Calhoun, V.D., 2019. Transient increased thalamic-sensory connectivity and decreased whole-brain dynamism in autism. NeuroImage 190, 191-204. https://doi.org/10.1016/j.neuroimage.2018.06.003

Glerean, E., Salmi, J., Lahnakoski, J.M., Jääskeläinen, I.P., Sams, M., 2012. Functional Magnetic Resonance Imaging Phase Synchronization as a Measure of Dynamic Functional Connectivity. Brain Connect. 2, 91-101. https://doi.org/10.1089/brain.2011.0068

Hasson, U., Nir, Y., Levy, I., Fuhrmann, G., Malach, R., 2004. Intersubject synchronization of cortical activity during natural vision. Science 303, 1634-40. https://doi.org/10.1126/science.1089506

Hutchison, R.M., Womelsdorf, T., Allen, E. a., Bandettini, P. a., Calhoun, V.D., Corbetta, M., Della Penna, S., Duyn, J.H., Glover, G.H., Gonzalez-Castillo, J., Handwerker, D. a., Keilholz, S., Kiviniemi, V., Leopold, D. a., de Pasquale, F., Sporns, O., Walter, M., Chang, C., 2013. Dynamic functional connectivity: Promise, issues, and interpretations. NeuroImage 80, 360-378. https://doi.org/10.1016/j.neuroimage.2013.05.079

Kang, J., Wang, L., Yan, C., Wang, J., Liang, X., He, Y., 2011. Characterizing dynamic functional connectivity in the resting brain using variable parameter regression and Kalman filtering approaches. NeuroImage 56, 1222-34. https://doi.org/10.1016/j.neuroimage.2011.03.033 
Kauppi, J.-P., Jääskeläinen, I.P., Sams, M., Tohka, J., 2010. Inter-subject correlation of brain hemodynamic responses during watching a movie: localization in space and frequency. Front. Neuroinformatics 4. https://doi.org/10.3389/fninf.2010.00005

Leonardi, N., Van De Ville, D., 2015. On spurious and real fluctuations of dynamic functional connectivity during rest. NeuroImage 104, 430-436. https://doi.org/10.1016/j.neuroimage.2014.09.007

Lindquist, M.A., Xu, Y., Nebel, M.B., Caffo, B.S., 2014. Evaluating dynamic bivariate correlations in resting-state fMRI: A comparison study and a new approach. NeuroImage 101, 531-546. https://doi.org/10.1016/j.neuroimage.2014.06.052

Nastase, S.A., Gazzola, V., Hasson, U., Keysers, C., 2019. Measuring shared responses across subjects using intersubject correlation. Soc. Cogn. Affect. Neurosci. 14, 667-685. https://doi.org/10.1093/scan/nsz037

Nummenmaa, L., Glerean, E., Viinikainen, M., Jääskeläinen, I.P., Hari, R., Sams, M., 2012. Emotions promote social interaction by synchronizing brain activity across individuals. Proc. Natl. Acad. Sci. U. S. A. 109, 9599-9604. https://doi.org/10.1073/pnas.1206095109

Park, H.-J., Friston, K., 2013. Structural and Functional Brain Networks: From Connections to Cognition. Science 342, 1238411-1238411. https://doi.org/10.1126/science.1238411

Power, J.D., Barnes, K.A., Snyder, A.Z., Schlaggar, B.L., Petersen, S.E., 2012. Spurious but systematic correlations in functional connectivity MRI networks arise from subject motion. NeuroImage 59, 2142-2154. https://doi.org/10.1016/j.neuroimage.2011.10.018

Raichle, M.E., MacLeod, A.M., Snyder, A.Z., Powers, W.J., Gusnard, D.A., Shulman, G.L., 2001. A default mode of brain function. Proc. Natl. Acad. Sci. U. S. A. 98, 676-82. https://doi.org/10.1073/pnas.98.2.676

Richardson, H., Lisandrelli, G., Riobueno-Naylor, A., Saxe, R., 2018. Development of the social brain from age three to twelve years. Nat. Commun. 9, 1-12. https://doi.org/10.1038/s41467-01803399-2

Rosenthal, G., Sporns, O., Avidan, G., 2017. Stimulus Dependent Dynamic Reorganization of the Human Face Processing Network. Cereb. Cortex 27, 4823-4834. https://doi.org/10.1093/cercor/bhw279

Silani, G., Lamm, C., Ruff, C.C., Singer, T., 2013. Right Supramarginal Gyrus Is Crucial to Overcome Emotional Egocentricity Bias in Social Judgments. J. Neurosci. 33, 15466-15476. https://doi.org/10.1523/JNEUROSCI.1488-13.2013

Simony, E., Honey, C.J., Chen, J., Lositsky, O., Yeshurun, Y., Wiesel, A., Hasson, U., 2016. Dynamic reconfiguration of the default mode network during narrative comprehension. Nat. Commun. 7, 12141. https://doi.org/10.1038/ncomms 12141

Teichert, T., Grinband, J., Hirsch, J., Ferrera, V.P., 2010. Effects of heartbeat and respiration on macaque fMRI: Implications for functional connectivity. Neuropsychologia 48, 1886-1894. https://doi.org/10.1016/j.neuropsychologia.2009.11.026

Zhang, Z.G., Fu, Z.N., Chan, S.C., Hung, Y.S., Motta, G., Di, X., Biswal, B.B., 2013. Adaptive window selection in estimating dynamic functional connectivity of resting-state fMRI, in: 2013 9th International Conference on Information, Communications \& Signal Processing. IEEE, pp. 1-4. https://doi.org/10.1109/ICICS.2013.6782935 
581 Table 1 Clusters with differential intersubject correlations of dynamic connectivity among the medial

582 visual, lateral visual, and supramarginal gyrus seeds. All clusters were thresholded at $\mathrm{p}<0.001$, and

583 cluster thresholded at $\mathrm{p}<0.0167(0.05 / 3)$ after family-wise error (FWE) correction using nonparametric

584 method.

\begin{tabular}{|c|c|c|c|c|c|c|}
\hline \multirow[b]{2}{*}{ cluster FWE } & \multirow[b]{2}{*}{ Voxel } & \multicolumn{3}{|c|}{ MNI Coordinates } & \multirow[b]{2}{*}{ Peak t } & \multirow[b]{2}{*}{ Label } \\
\hline & & $\mathrm{x}$ & $\mathrm{y}$ & $\mathrm{z}$ & & \\
\hline \multicolumn{7}{|c|}{ Medial visual > (lateral visual + supramarginal) } \\
\hline$<0.001$ & 514 & -12 & -100 & -4 & 7.85 & Occipital pole \\
\hline$<0.001$ & 372 & 24 & -88 & -4 & 6.80 & Right inferior occipital gyrus \\
\hline 0.001 & 106 & -51 & 11 & 23 & 5.87 & Left precentral gyrus \\
\hline \multicolumn{7}{|c|}{ Lateral visual > (Medial visual + supramarginal) } \\
\hline 0.016 & 78 & 48 & -70 & 8 & 7.03 & Right inferior occipital gyrus \\
\hline 0.013 & 86 & 15 & -79 & -4 & 5.64 & Lingual gyrus \\
\hline \multicolumn{7}{|c|}{ Supramarginal > (medial visual + lateral visual) } \\
\hline$<0.001$ & 1168 & 3 & -40 & 44 & 9.12 & Precuneus \\
\hline 0.002 & 237 & -12 & 50 & 14 & 7.41 & Medial superior frontal gyrus \\
\hline 0.006 & 122 & -30 & -4 & 44 & 6.90 & Left middle frontal gyrus \\
\hline 0.003 & 175 & 18 & 50 & 44 & 6.44 & Right middle frontal gyrus \\
\hline
\end{tabular}

585

MNI, Montreal Neurological Institute 